\title{
Ophthalmic Manifestations of Vitamin A and D Deficiency in Two Autistic Teenagers: Case Reports and a Review of the Literature
}

\author{
Emma Duignan $^{\mathrm{a}}$ Paul Kenna $^{\mathrm{a}}$ Rosemarie Watson $^{\mathrm{b}}$ Susan Fitzsimon ${ }^{\mathrm{c}}$ \\ Donal Brosnahan a, b \\ ${ }^{a}$ Royal Victoria Eye and Ear Hospital, ${ }^{b}$ Our Lady's Children's Hospital, and ${ }^{c}$ Mater Private \\ Hospital, Dublin, Ireland
}

\section{Key Words}

Vitamin A · Autism · Phrynoderma $\cdot$ Bitot's spots $\cdot$ Xerophthalmia

\begin{abstract}
We describe the cases of 2 autistic children with ophthalmic and systemic manifestations of vitamin A deficiency due to food faddism. Although vitamin A deficiency is common in the developing world, reports in developed societies are rare. Our patients presented over a 1year period. The patients were 14 and 13 years old at the time of presentation and were both found to have marked features of vitamin A deficiency related to unusual dietary habits. Anterior segment signs of xerophthalmia were present in both patients. In addition, patient 1 showed evidence of a rod-predominant retinopathy, which resolved with vitamin A supplementation. Due to its rare occurrence, hypovitaminosis A must be highlighted and anticipated in this cohort.

(c) 2015 S. Karger AG, Basel
\end{abstract}

\section{Introduction}

Vitamin A deficiency is common in the developing world but has historically been rare in developed countries [1]. Despite this, a small number of patients with psychiatric conditions such as autism, food phobia and anorexia nervosa have been diagnosed with hypovitaminosis A related to food faddism [2-10]. We describe the cases of 2 teenage patients with a background of moderate autistic spectrum disorder who were diagnosed with a multi- 
Duignan et al.: Ophthalmic Manifestations of Vitamin A and D Deficiency in Two Autistic Teenagers: Case Reports and a Review of the Literature

vitamin deficiency, including vitamin A. Their ophthalmic and systemic manifestations resolved with vitamin replacement therapy.

\section{Case 1}

A 14-year-old boy was admitted to the emergency department of a pediatric hospital complaining of bilaterally decreased visual acuity for 5 days and a persistent rash on the upper limbs for 10 weeks. His ophthalmic history included bilateral chalazia and blepharitis. His psychiatric history included a moderate autism spectrum disorder diagnosed at the age of 2, necessitating dedicated schooling. At presentation, his best-corrected visual acuity was $20 / 25$ in both eyes. The optic discs were mildly swollen bilaterally, and no relative afferent pupillary defect was recorded. The child was noted to have an increased BMI, and a follicular, erythematous rash was present, affecting predominantly the extensor surface of the upper limbs (fig. 1a). The initial differential diagnosis included optic neuritis and herpes zoster, given the vesicular appearance of the rash. The child was admitted and treated with intravenous methylprednisolone as well as intravenous acyclovir by the admitting team. MRI and CT of the brain and the orbits as well as a lumbar puncture were performed, all of which were within normal limits. Flash and pattern-reversal visual evoked responses were performed monocularly in each eye, and responses were within the normal range.

Two days after admission, the patient was seen by the ophthalmology team and was found to have a left relative afferent pupillary defect, bilaterally swollen optic discs and a featureless, dry conjunctiva with Bitot's spots (fig. 2). Vitamin A, $B_{12}$ and D levels were recorded. His vitamin A level was undetectable $(<0.1 \mathrm{ng} / \mathrm{ml})$, the vitamin $B_{12}$ level was borderline $(150 \mathrm{pg} / \mathrm{ml})$, and the vitamin D level was found to be insufficient $(32 \mathrm{ng} / \mathrm{ml})$. An electroretinogram (ERG) showed a marked attenuation of $A$ and $B$ waves in rod-isolated sequences (fig. 3), even though the patient did not describe nyctalopia and the parents denied observing any behavior suggestive of night blindness. Single flash and $30-\mathrm{Hz}$ cone-isolated responses remained normal.

On further questioning, the mother of the teenager attested to his severely restricted diet consisting almost entirely of bread and fried potatoes. A diagnosis of hypovitaminosis A was made with the presence of xerophthalmia, retinopathy and phrynoderma, a characteristic rash (fig. 3).

Vitamin replacement therapy was commenced with daily oral vitamin A supplementation of 25,000 IU per day (Vitasorb A, Biocare, Birmingham, UK), and an attempt to increase dietary vitamin intake. One month later, the phrynoderma had resolved, leaving pale, follicular scarring (fig. 1b). Repeat ERG was within normal limits, and the patient's vision was normalizing. His parents reported an increase in alertness and school performance.

\section{Case 2}

A 13-year-old boy, diagnosed with a moderate autistic spectrum disorder, attended the outpatient department with a 6-week history of bilateral decreased visual acuity, redness of the eyes, light sensitivity and an inability to keep his eyes open. The parents noted deteriorating alertness at home. He was commenced on a trial of topical steroids and lubricants with an initial diagnosis of severe adenoviral infection. He underwent an examination under anesthesia which showed corneal and conjunctival xerosis. A conjunctival biopsy was dif- 
Duignan et al.: Ophthalmic Manifestations of Vitamin A and D Deficiency in Two Autistic Teenagers: Case Reports and a Review of the Literature

fusely abnormal, with squamous metaplasia of the conjunctival epithelium, submucosal inflammation and marked keratinization (fig. 4).

Serum vitamin A and D levels were obtained and showed an insufficient vitamin D level $(<15 \mathrm{ng} / \mathrm{ml})$ and an undetectable level of vitamin A. A malabsorptive cause was eliminated, and the patient's diet was found to be severely restricted. Oral vitamin supplementation was commenced and an improvement was noted almost immediately, with the complete resolution of his symptoms within 1 week.

\section{Discussion}

Vitamin A deficiency is a leading cause of childhood blindness worldwide, though historically rare in developed countries. It has been reported in the psychiatric literature that it can be related to food faddism in cases of autism [2-8] and eating disorders $[9,10]$, often related to a restricted diet, high in fried potatoes. There are also reports of this deficiency due to malabsorption from gastric bypass surgery [11], primary biliary cirrhosis [8] and chronic giardiasis [12]. There is a possibility that we may see increasing numbers of patients with hypovitaminosis A related to alcoholic liver disease in years to come [13].

There have been 7 cases of vitamin A deficiency in autistic children reported in the literature since 1993 (table 1). Ocular manifestations of vitamin A deficiency in this cohort include xerophthalmia, corneal ulceration, retinal pigment epithelial changes and optic atrophy. Corneal and conjunctival xerosis are the most commonly reported manifestations of the condition. Bitot's spots are a pathognomonic sign of vitamin A deficiency and indicate conjunctival xerosis with a deposition of keratin in the conjunctival epithelium. The severity of the corneal disease in some cases warrants penetrating keratoplasty, for example that of Steinemann and Christiansen [2]. Fundal pathology is more rarely reported. Yellow flecks at the level of the retinal pigment epithelium were reported by Steinemann and Christiansen. Although no ERG was performed prior to replacement with vitamin A, scotopic and photopic ERG were within normal limits after treatment. McAbee et al. [3] report the case of an autistic child developing bilateral optic atrophy secondary to vitamin A deficiency. Although 2 patients in this group reported nyctalopia, the rod-predominant retinopathy recorded in our patient has not previously been demonstrated on ERG. Interestingly, although our patient did display a rod retinopathy, which responded to vitamin A replacement, there was no evidence of any difficulty with night vision. We also note the parents' description of a general decline in behavior in both patients, which was ameliorated by vitamin replacement therapy. It is unclear whether this change in behavior is related to the patients' experience of their symptoms or a cognitive deficit secondary to malnutrition. The World Health Organization (WHO) recommendations for the treatment of vitamin A deficiency in the context of xerophthalmia advise an initial, immediate oil-based oral dose of 200,000 IU, followed by the same dose on days 2 and 14. This must be supplemented by 4- to 6-monthly doses of 200,000 IU [14].

Phrynoderma, meaning 'toad skin' in Greek, is a papular perifollicular eruption caused by follicular hyperkeratosis, which is historically linked to vitamin A deficiency. It was first reported by Lucius Nicholls in 1933 [15], who observed the characteristic rash in malnourished African laborers. Other dermatological manifestations of vitamin A deficiency include blepharitis [16], noted in our first patient, and cheilitis.

In conclusion, although vitamin deficiencies in children are rare in developed societies, a low threshold for vitamin level testing should be undertaken in autistic spectrum disorder cases, highlighting the importance of enquiring about dietary habits. If vitamin deficiency is 
Duignan et al.: Ophthalmic Manifestations of Vitamin A and D Deficiency in Two Autistic Teenagers: Case Reports and a Review of the Literature

found, patients should also be investigated for malabsorption and hepatic pathology. Treatment with replacement vitamins in the appropriate form can have a rapid therapeutic effect. It should also be emphasized that appropriate counseling of the patients' parents, particularly with regard to a healthy diet and continued supplementation of vitamins, is essential to prevent a recurrence of hypovitaminosis.

\section{Disclosure Statement}

The authors have no financial interests to declare.

\section{References}

1 World Health Organization: Global Prevalence of Vitamin A Deficiency in Populations at Risk 1995-2005. WHO Global Database on Vitamin A Deficiency. Geneva, World Health Organization, 2009.

-2 Steinemann TL, Christiansen SP: Vitamin A deficiency and xerophthalmia in an autistic child. Arch Ophthalmol 1998;116:392-393.

-3 McAbee GN, Prieto DM, Kirby J, Santilli AM, Setty R: Permanent visual loss due to dietary vitamin A deficiency in an autistic adolescent. J Child Neurol 2009;24:1288-1289.

-4 Lewis CD, Traboulsi EI, Rothner AD, Jeng BH: Xerophthalmia and intracranial hypertension in an autistic child with vitamin A deficiency. J Pediatr Ophthalmol Strabismus 2010:1-3.

5 Tanoue K, Matsui K, Takamasu T: Fried-potato diet causes vitamin A deficiency in an autistic child. JPEN J Parenter Enteral Nutr 2012;36:753-755.

6 Clark JH, Rhoden DK, Turner DS: Symptomatic vitamin A and D deficiencies in an eight-year-old with autism. JPEN J Parenter Enteral Nutr 1993;17:284-286.

7 Uyanik O, Dogangun B, Kayaalp L, Korkmaz B, Dervent A: Food faddism causing vision loss in an autistic child. Child Care Health Dev 2006;32:601-602.

-8 Lin P, Fintelmann RE, Khalifa YM, Bailony MR, Jeng BH: Ocular surface disease secondary to vitamin A deficiency in the developed world: it still exists. Arch Ophthalmol 2011;129:798-799.

-9 Cooney TM, Johnson CS, Elner VM: Keratomalacia caused by psychiatric-induced dietary restrictions. Cornea 2007;26:995-997.

10 Velasco Cruz AA, Attie-Castro FA, Fernandes SL, Cortes JF, de Tarso PP-FP, Rocha EM, et al: Adult blindness secondary to vitamin A deficiency associated with an eating disorder. Nutrition 2005;21:630-633.

-11 Zalesin KC, Miller WM, Franklin B, Mudugal D, Rao Buragadda A, Boura J, et al: Vitamin A deficiency after gastric bypass surgery: an underreported postoperative complication. J Obes 2011;2011.

12 Girard C, Dereure 0, Blatiere V, Guillot B, Bessis D: Vitamin A deficiency phrynoderma associated with chronic giardiasis. Pediatr Dermatol 2006;23:346-349.

13 McLaughlin S, Welch J, MacDonald E, Mantry S, Ramaesh K: Xerophthalmia - a potential epidemic on our doorstep? Eye (Lond) 2014;28:621-623.

14 WHO, UNICEF, IVACG Task Force: Vitamin A Supplements: A Guide to Their Use in the Treatment and Prevention of Vitamin A Deficiency and Xerophthalmia, ed 2. Geneva, World Health Organization, 1997. http://whqlibdoc.who.int/publications/1997/9241545062.pdf (accessed January 6, 2014).

15 Nicholls L: Phrynoderma: A condition due to vitamin deficiency. Indian Med Gaz 1933;68:681-687.

16 Chen L, Chen X, Xiang Q, Zheng Y, Pi L, Liu Q, et al: Prevalence of low serum vitamin A levels in young children with chalazia in southwest China. Am J Ophthalmol 2014;157:1103-1108.e2. 
Case Reports in

Ophthalmology

\begin{tabular}{l|l}
\hline \multicolumn{2}{l|}{ Case Rep Ophthalmol 2015;6:24-29 } \\
\hline DOI: $10.1159 / 000373921$ & $\begin{array}{l}\text { C } 2015 \text { S. Karger AG, Basel } \\
\text { www.karger.com/cop }\end{array}$ \\
\hline
\end{tabular}

Duignan et al.: Ophthalmic Manifestations of Vitamin A and D Deficiency in Two Autistic Teenagers: Case Reports and a Review of the Literature

Table 1. Reported cases of vitamin A deficiency secondary to dietary restriction in autistic children to date

\begin{tabular}{llll}
\hline Author & Age & Manifestation & Diet \\
\hline Clark et al. [6] & 8 & Xerophthalmia, corneal ulceration & Fried potatoes \\
\hline $\begin{array}{l}\text { Steinemann and Christian- } \\
\text { sen [2] }\end{array}$ & 5 & $\begin{array}{l}\text { Corneal ulceration, descemetocele, } \\
\text { peripheral RPE 'yellow flecks', nyctalopia }\end{array}$ & $\begin{array}{l}\text { Bacon, blueberry } \\
\text { muffins }\end{array}$ \\
\hline Uyanik et al. [7] & 8 & Xerophthalmia, corneal ulceration & Fried potatoes \\
\hline McAbee et al. [3] & Adolescent & Optic atrophy & Restricted \\
\hline Lewis et al. [4] & 10 & Xerophthalmia & Restricted \\
\hline Lin et al. [8] & 9 & Xerophthalmia, optic atrophy, nyctalopia & Fried potatoes \\
\hline Tanoue et al. [5] & 5 & Xerophthalmia & Fried potatoes \\
\hline
\end{tabular}

$\mathrm{RPE}=$ Retinal pigment epithelium.
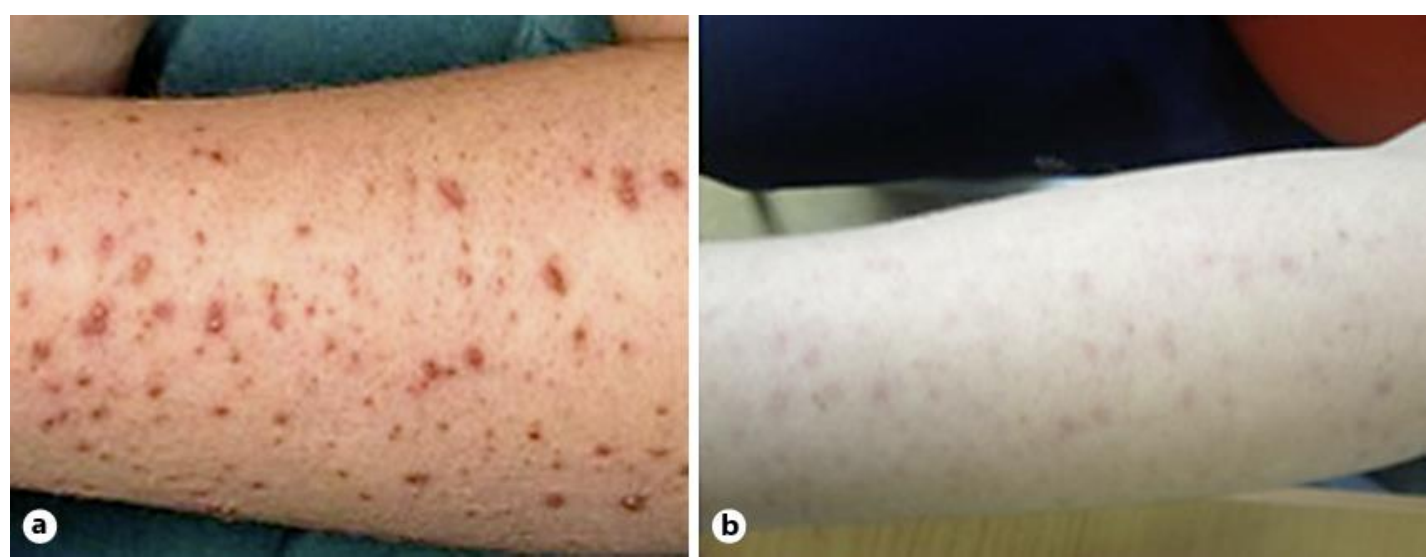

Fig. 1. Phrynoderma before treatment (a) and subtle follicular scarring after treatment (b).

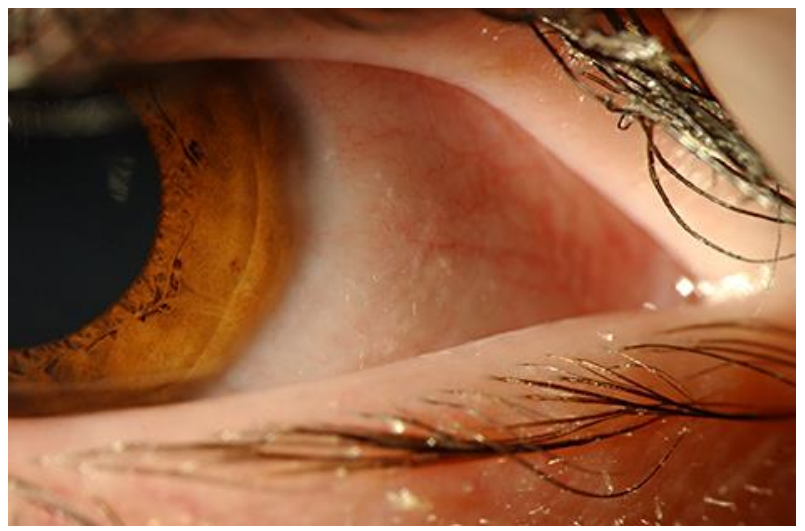

Fig. 2. Bitot's spots on the temporal conjunctiva (patient 1). 


\section{Case Reports in \\ Ophthalmology}

\begin{tabular}{l|l}
\hline \multicolumn{2}{l|}{ Case Rep Ophthalmol 2015;6:24-29 } \\
\hline DOI: $10.1159 / 000373921$ & $\begin{array}{l}\text { C } 2015 \text { S. Karger AG, Basel } \\
\text { www.karger.com/cop }\end{array}$ \\
\hline
\end{tabular}

Duignan et al:: Ophthalmic Manifestations of Vitamin A and D Deficiency in Two Autistic Teenagers: Case Reports and a Review of the Literature

\section{Pre Vitamin A Treatment}

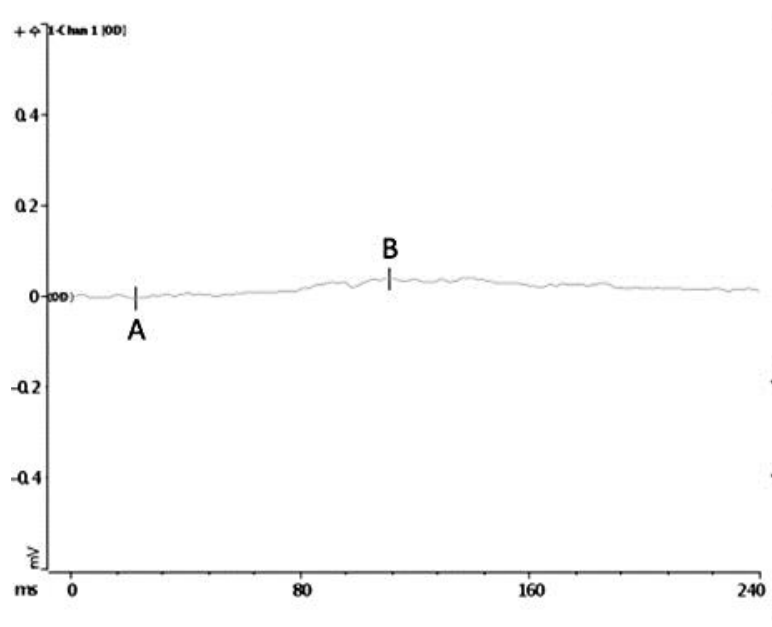

5-weeks Post Vitamin A Treatment

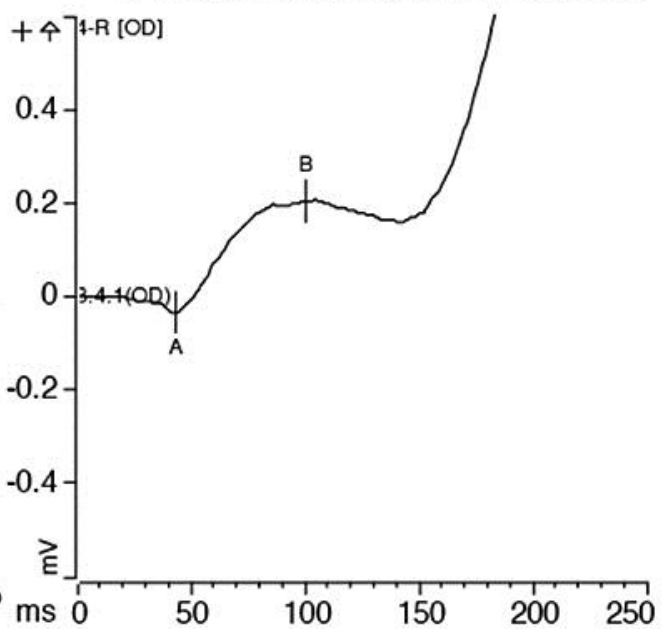

Fig. 3. Scotopic ERG pre- and post-vitamin A replacement therapy.
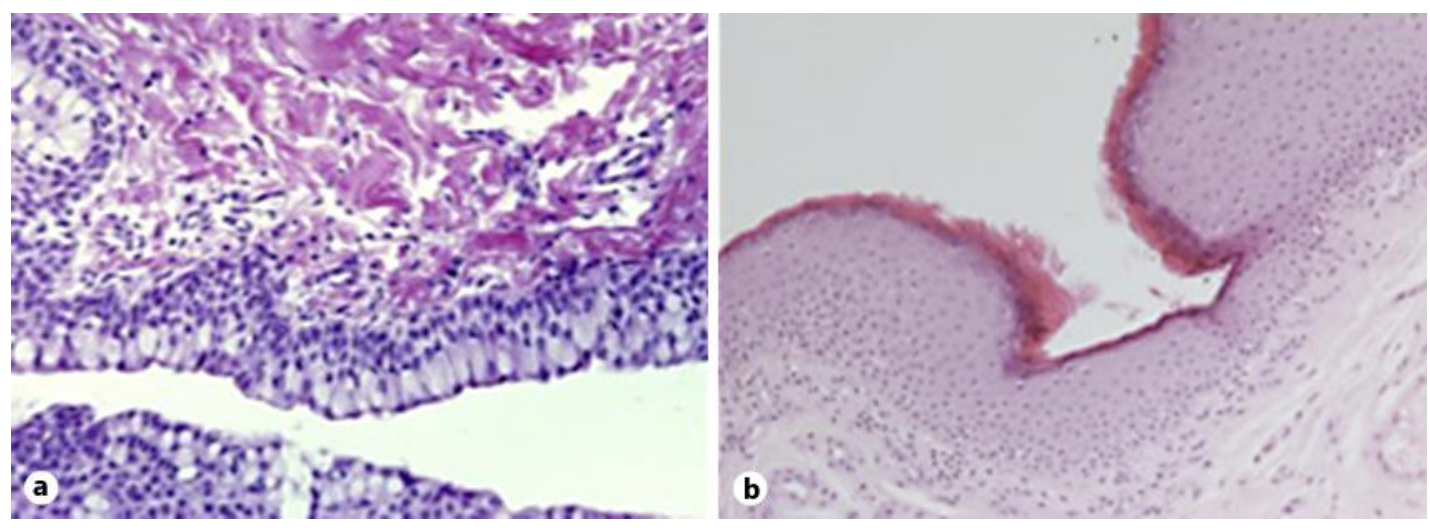

Fig. 4. Normal conjunctival epithelium (a) and conjunctival epithelium of patient 2 (b) with squamous metaplasia and keratinization of conjunctival epithelium. 